\title{
Uniform and Non-Uniform Image Enlargement Based on the Window Kernel
}

\author{
I Komang Somawirata ${ }^{*}$, Bambang Prio Hartono, Teguh Herbasuki, and Aryuato Soetedjo \\ Electrical Engineering, Faculty of Industrial Technology, National Institute of Technology (ITN \\ Malang), Karanglo km 2, Malang 65145 Indonesia.
}

\begin{abstract}
Several different of image resizing methods have been proposed. However, resizing image in different ratio produces non proportional feature image enlarged and the enlarged image in different ratio with retaining the salient content is limited. This paper proposes an image enlargement method with edge improvement and inpainting method. This method consists of two steps. First, the image enlargement method is used for enlarging an image to the minimum size of the target image. Second, image inpainting method is used for obtaining the full-size image. We have evaluated the proposed method for enlarging an image uniformly and it compares with the typical of image enlargement methods. The uniform image enlarged and image enlarged in different ratio using the proposed method have been presented.
\end{abstract}

Key words: Image enlargement, inpainting, window kernel

\section{Introduction}

Image enlargement is changing small size image to bigger size. Image enlargement can be done in two ways. There are using hardware or optical zoom-in and software or digital zoom-in. Image enlargement using hardware is a lot more expensive than using software. Image enlargement has been applied to numerous fields such as medical photo results, photo satellite, digital TV, digital camera etc. Currently, many sizes and ratios of display have been presented. The ratios of display size are ranging from 3:2, 4:3, 5:4, 16:9, 16:10, 17:9 and 21:9 respectively for the width and height. So that, the image enlargement method for enlarging images in uniform and non-uniform is needed.

There are several methods that have been developed to refine the performance of classic methods such as, image enlargement methods in [1-5]. The method [1] proposed image enlargement using weighing method known as WLE method. This interpolation method explains interpolation in three different conditions: interpolation between two horizontal pixels, interpolation between two vertical pixels, and interpolation among four pixels. WLE method does not provide good result especially in enlarging images whose scale factor is bigger than two. However, WLE method can be easily computed so it is very convenient to apply to hardware. The image enlargement method using edge-direction has been proposed in $[2,3]$. The recovery of sharp edges on high-resolution (HR) image using reverse diffusion interpolation (RDI) [2] is capable for reducing blurred edge image. The blur edges

*Corresponding author: kmgsomawirata@,lecturer.itn.ac.id 
image is caused by low frequency. It could happen during the application of low-pass-filter during the data-acquisition process. RDI uses nine pixels-samples with $3 \times 3$ windows. Elements of matrix $3 \times 3$ windows are ordered using rank-order-statistic. Partial differential equation is used to obtain the value of the highest gradient direction to produce the reverse diffusion process. RDI method is no filtering in homogeneous regions, and no extra parameter is needed to characterize an edge. The image enlargement based on regularity on the geometric is presented by [3]. This method is called by new edge-directed interpolation (NEDI). NEDI method uses covariance-based adaptive interpolation especially in edge and pixels near of edge. They use bilinear interpolation for non-edge pixels. So that, the scale factor for NEDI method is only for even value. The method [4] proposes a frequency domain method which based on low frequency aliasing-free part. This method is called by frequency domain approach (FDA). FDA uses bicubic interpolation for image reconstruction. The low-frequency aliasing-free part of the image is used to estimate planar rotation and translation parameters. Image enlargement based on windows kernel by applying the pyramid method has been done in [5]. This method is called with PWK. The PWK method is also easy to be implemented on image retargeting. Target image size is not only in the same ratio, but also in the different ratio can be handled. In addition, PWK can be implemented in the variety of shapes and sizes of the target image. However, the methods [1-5] are only good for uniform enlarging. When the scaling factor for high and width are different, then non proportional salient features are obtained.

Recently, many researchers especially down-scale on image retargeting start to focus on dealing the visual salience, while suppressing non-salient regions [6]. Seam carving method [7] is a novel content-aware resizing scheme. This method uses gradient magnitude for measuring the image importance. However, the visual distortions often occur when it is used to up-scale image.

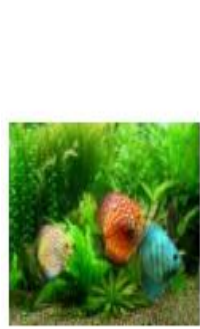

(a)

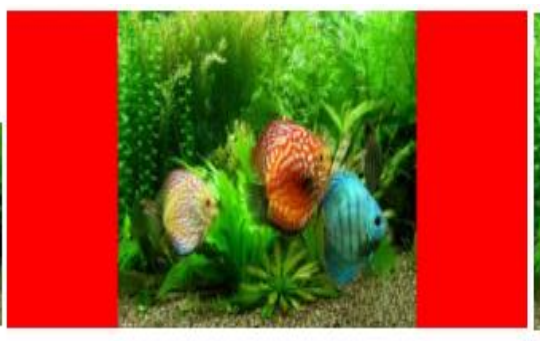

(b)

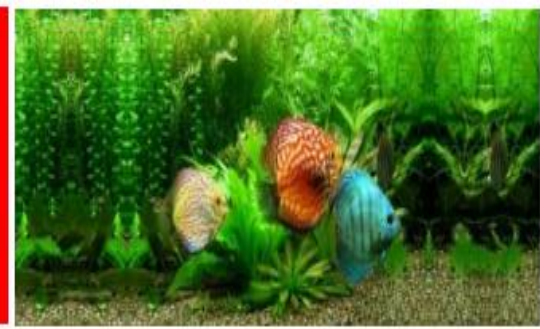

(c)

Fig. 1. The combination of image enlargement and inpainting methods. (a) Source image size. (b) Source image is enlarged to the minimum size of target image. (c) Full size image is obtained by inpainting method.

Currently, many researcher adopt the seam carving method for image scaling which retains the salient features of the image. The method [8] improves the warping-based image retargeting by adding the line constraints after applying original retargeting process. Image enlargement which is only based on seam carving that results the same size of the salient feature with the salient feature in the source image. So that, the combination of seam carving with image enlargement method [9] has resulted proportional salient feature image enlarged. This method requires iterations until the target size image is obtained. The method [9] is improved by [10], which is without iteration process. However, the energy gradient is not optimal for guiding the slicing process. The energy gradient is improved by salient feature energy which is adopted from [11]. The method [12] uses salient feature 
energy for guiding the slicing process. So, the slicing process is optimal for selecting the non-salient feature.

Image enlargement based on seam carving and slicing fail for enlarging an image, if the salient feature in an image is connecting from left to right sides of image. To overcome the limitation of existing methods, we propose the image retargeting especially for image enlarging based on the uniform image enlargement and image in painting methods. The image upscaling method uses the image enlargement method based on window kernel with edge improvement.

\section{Proposed method}

Our method is a combination between image enlargement and image inpainting methods. The image enlargement method is based on window kernel method with edge improvement, and inpainting method is also using window kernel method. Detailed explanation about these methods will be explained in the following subsection.

\subsection{Image enlargement based on window kernel and edge improvement}

The source image or low-resolution (LR) image is symbolized by I with the size $N \times M(N$ and $M$ are height and width of LR image). The size of an image enlarged ( $\overline{\mathbf{I}})$ is obtained by multiplying the size of the LR image (I) with scale factor $(S)$. In this case, the image $\hat{\mathbf{I}}$ is still empty (no pixel), then we fill each coordinate in image $\mathbf{I}$ by pixels which is calculated by Equation 1.

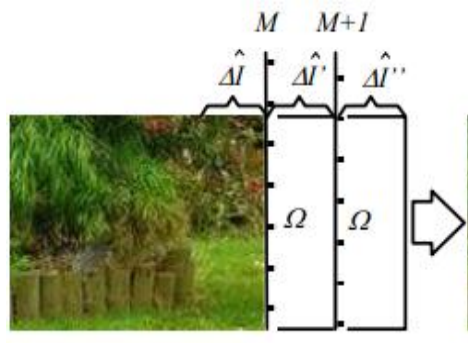

(a)

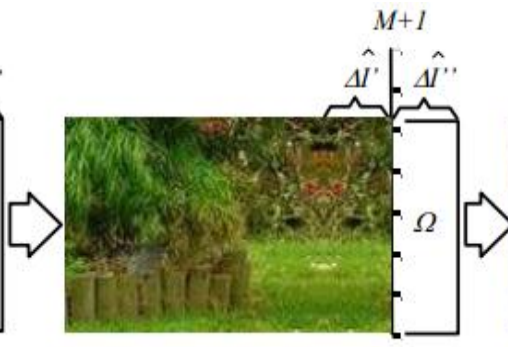

(b)

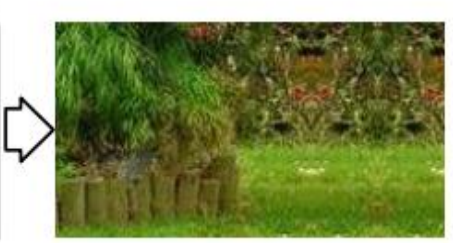

(c)

Fig. 2. Image enlarged based on inpainting with multi-mirroring method. (a) Source image that will be enlarged by multi-mirroring method ( $M$ and $M+1)$. (b) The first mirroring has done. (c) The second mirroring has done

Equation 1 is adopted from bilinear interpolation formula. However, the weight window is not only in linear weighting, but also with weighting which is based on edge direction. An image $\hat{\mathbf{I}}$ is uniformly image enlarged with $\widehat{N} \times \widehat{M}(\widehat{N}=N S$ and $\widehat{M}=M S)$.

$$
\hat{\mathbf{I}}_{x y}=\sum_{j=1}^{2} \sum_{i=1}^{2} W_{i j}^{P} W_{i j}^{W}
$$

$W_{i j}^{P}$ is the element of sample pixel window $\mathbf{W}^{\mathrm{P}}$ and $W_{i j}^{W}$ is the element of weight window $\mathbf{W}^{\mathbf{W}} . \hat{I}_{x \hat{y}}$ is element of HR image. $\hat{x}$ and $\hat{y}$ are height and width coordinates of HR image. 


\subsection{Image inpainting}

As in Figure 1(b), inpainting method is used for completing the red areas in the left side $\left(\Omega^{\mathbf{L}}\right)$ and right side $\left(\boldsymbol{\Omega}^{\mathbf{R}}\right)$ of target image. We use an inpainting method like a multimirroring process. The $\boldsymbol{\Omega}^{\mathbf{L}}$ and $\boldsymbol{\Omega}^{\mathbf{R}}$ are element of image $\hat{\mathbf{I}} \notin \widehat{\Omega}$, where $\widehat{\boldsymbol{\Omega}}$ is blocking area in image $\hat{\mathbf{I}}$.

However, the $\widehat{\Omega}$ can be manually or automatically selected. The mirroring process just as wide $\Delta \hat{\mathbf{I}}$, and it is continuing to repeat mirroring the $\Delta \hat{\mathbf{I}}$ until the full size of target image obtained. $\Delta \hat{\mathbf{I}}$ is the width size of mirroring process. If in the mirroring process contains a region of $\widehat{\Omega}$, then we will change it with a pixel which is obtained from the close area of $\widehat{\Omega}$.

The multi-mirroring method can also be implemented for inpainting an image as in the inpainting section on Figure 3. The multi-mirroring method is used for completing the big hole or blank area in the image.

\subsection{Proportional salient feature image enlarged}

Proportional salient features image enlargement is obtained, if only the scale factor for the height and width in the same value. While the salient features will not be proportional, if the scale factor for the height and width are not equal. To solve the problem of magnification image at the different ratio, we do it with two stages, such as illustrated in Figure 1. First, source image (Figure 1(a)) is enlarged uniformly to the smallest size of the target image as in Figure 1(b). Second, the inpainting method is applied to get the full size image as in Figure 1(c).

In this paper, we explain two solutions for obtaining an enlarged image in the different ratio. First, Enlarge an image in Figure 1(b) uses multi-mirroring method as illustrated in Figure 2. In this example, an image is mirrored to right side by two times $\Delta \widehat{I}$. The first and second mirroring results are shown in Figure 2(b) and Figure 2(c), respectively. The second solution of image enlarged in different ratio uses a method that is illustrated in Figure 3. In this method, we separate the region of interest (ROI) in an image. For automatically ROI selected, we use saliency image detection that has been proposed in [11], and we symbolize the saliency image with $\mathbf{I}_{\mathbf{s}}$. However, the saliency detection in $\mathbf{I}_{\mathbf{s}}$ is not covering full region of ROI. So that, we are convolving $(*)$ the $\mathbf{I}_{\mathbf{s}}$ with a circular averaging filter $\left(\mathbf{H}_{\mathbf{c}}\right)$ with radius equal to ten as in Equation 2. By this solution, we can increase the region of ROI.

$\hat{\mathbf{I}}_{5}=\mathbf{I}_{5} * \mathbf{H}_{c}$

So that, the salient feature map $\left(\mathbf{F}_{\mathbf{s}}\right)$ is obtained by threshold $(\tau)$ the value of feature image $\hat{\mathbf{I}}_{s}$, as in Equation 3. We set the threshold value slightly bigger than zero.

$\mathbf{F}_{5}= \begin{cases}1 & \text { if } \hat{\mathbf{I}}_{5}>\tau \\ 0 & \text { others }\end{cases}$

The salient feature map $\left(\mathbf{F}_{\mathbf{s}}\right)$ is a binary image with high-intensity values equal to 1 and low intensity value equal to 0 , where 1 and 0 represent salient and a non salient feature of image as shown in Figs. 6(e) and (g). The ROI is separated by multiplying the salient feature map and image $\mathbf{\mathbf { I }}$, as in Equation 4, while the non-ROI is obtained by multiplying the complement of the salient feature map with image $\mathbf{I}$, as in Equation 5. 


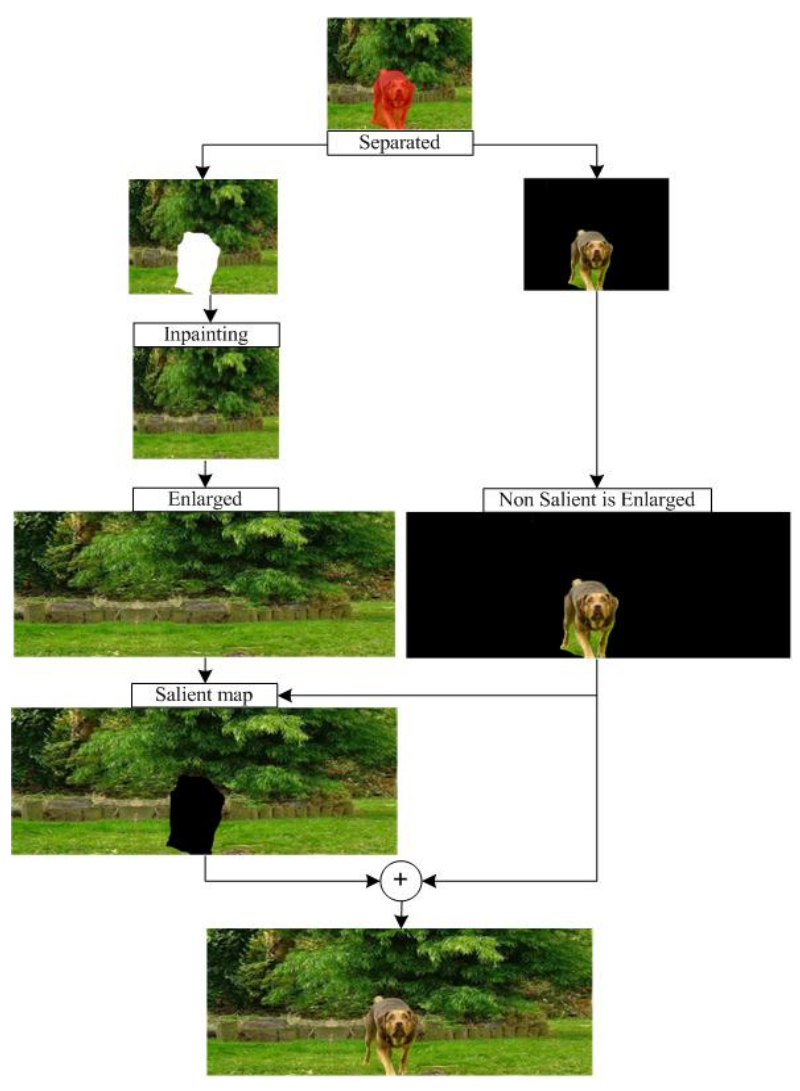

Fig. 3. Flow diagram of image enlargement in different ratio.

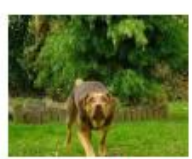

(a)

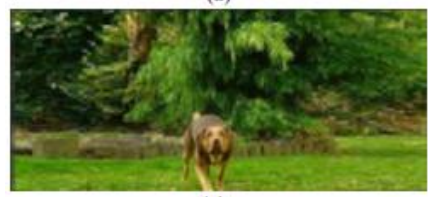

(c)

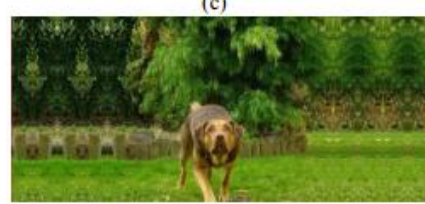

(e)

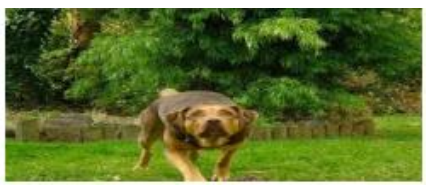

(b)

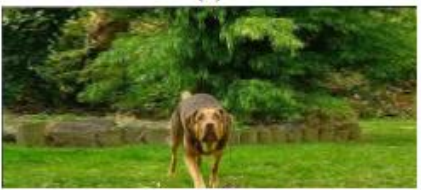

(d)

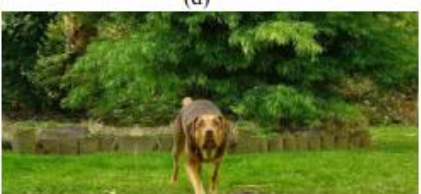

(f)

Fig. 4. Image enlargement in different ratio. (a) Source image. (b) The result of image enlargement based on window kernel and edge improvement. (c) The result of seam-carving method [7]. (d) The result of method [12]. (e) The result of multi-mirroring method. (f) Proposed method. 


$$
\begin{aligned}
& \mathbf{F}_{\mathrm{I}}^{5}=\hat{\mathbf{I}} \times \mathbf{F}_{5} \\
& \mathbf{F}_{\mathrm{I}}^{n}=\hat{\mathbf{I}} \times \overline{\mathbf{F}_{5}}
\end{aligned}
$$

where, $\mathbf{F}_{\mathrm{I}}^{5}$ and $\mathbf{F}_{\mathrm{I}}^{\mathbf{n}}$ are ROI and non-ROI image respectively. Overline ( ${ }^{\mathbf{z}}$ ) is NOT operator.

As in Figure 3, an image without ROI (the blank region) is inpainted by using the multimirroring method. The blank area is filled with pixels based on multi mirroring process start from left to right and right to left, until all empty areas filled with pixels. After the blank area is filled with pixels, this image is enlarged to different ratio, and then it is continued to make the ROI map. Finally, the image enlarged with maintained ROI is obtained by summing two images, there are an enlarged image with ROI map and an image which only contains ROI, as shown in Figure 3.

\section{Experimental result}

In this experiment, the proposed method is tested for uniform and nonuniform image enlargement. For uniform image enlargement, we evaluate the image enlargement result using PSNR and we use seven samples image such as baboon, boat, buterfly, clown, lena, peppers and parrot. These image resize to smaller size using nearest-neighbor method. The sizes of images which would be enlarged were quarter of the standard image size. After that, we enlarge the small image size using all methods by the scale factor equal to four to become the standard image size. We use four comparison methods such as bi-linear interpolation (BLI), NEDI [3], RDI [2] and WLE [1].

Table 1 shows the image quality evaluation using PSNR by a scale factor equal to four. The PSNR values of proposed method show bigger than the comparison method. This means that our method is better than the comparison method.

For non-uniform image enlarged, Figure 4 shows the comparison results of five kind image enlargement method. Figure 4 (a) is source image that will be enlarged to different ratio, and the target ratio is 21:9.

Table 1 PSNR result of four times image enlarged.

\begin{tabular}{|l|l|l|l|l|l|l|l|}
\hline \multirow{2}{*}{ Methods } & \multicolumn{7}{|c|}{ Image samples } \\
\cline { 2 - 8 } & Baboon & Boat & Buterfly & Clown & Lena & Peppers & Parrot \\
\hline BLI & 18.42 & 21.90 & 16.81 & 19.53 & 23.66 & 25.66 & 21.32 \\
\hline $\begin{array}{l}\text { NEDI } \\
{[3]}\end{array}$ & 17.54 & 19.57 & 15.12 & 16.90 & 20.73 & 21.96 & 19.00 \\
\hline RDI [2] & 18.30 & 21.74 & 16.70 & 19.30 & 23.41 & 25.43 & 21.09 \\
\hline WLE [1] & 18.4 & 22.25 & 16.72 & 19.67 & 24.41 & 26.66 & 21.58 \\
\hline $\begin{array}{l}\text { Proposed } \\
\text { Method }\end{array}$ & 20.14 & 24.34 & 18.87 & 22.60 & 27.20 & 29.85 & 23.99 \\
\hline
\end{tabular}

Figure 4(b) show the result of image enlarged using the image enlargement method based on window kernel and edge improvement. Figure 4(c) shows the image enlarged using a seam carving method that has been improved, Figure 4(d) the results of method [12], Figure 4(e) the results of multi-mirroring method and Figure 4(f) the result of the proposed method with a combination between "image enlargement method based on 
window kernel and edge improvement" and an image inpainting method based on multimirroring process.

Image enlargement in different ratio using scaling method produces non-proportional feature enlarged compared with their original image feature as in Figure 4(b). While, the seam carving method on Figure 4(c) produces the same size of the salient feature with the salient feature in the source image and it produces non-proportional feature enlarged, especially on the background of the image. Figure 4(d) shows a result of method [12]. In this result, only the slice region on left and right sides of ROI are enlarged to target image size. The image slicing direction is guided by feature energy of the image and the image slicing starts from top to bottom. So, the non ROI image is not uniformly enlarged to the wide size of the target image.

\section{Conclusion}

In this paper, we have proposed an image enlargement method for uniform and nonuniform image enlarging. The method is a combination between image enlargement method with edge direction based on the window kernel and image inpainting method. For uniform image enlarged, we only use image enlargement method with edge direction based on the window kernel, while for enlarging an image in different ratio (non-uniform enlarged), we combine it with image inpainting method, as in the following steps: first, the source image is enlarged to the minimum size of the target image. Second, the inpainting method is used for completing the empty areas. The proposed method has been tested for enlarging an image in uniform and non-uniform image enlarged. We have evaluated the uniform image enlarged result using PSR and The PSNR value of proposed method always bigger than the comparison method. The image enlarged in different ratio has been presented.

For the next research, we have plans to solve the problem of image enlargement, especially for different ratios. We will improve the inpainting method and salient feature detection.

\section{References}

1. T. Aso, N. Sutake, T. Yamakau. A Fast and Accurate Image Enlargement Algorithm Employing a Weighted Sum of Linear Extrapolations. Proceedings World Automation Congress 2004, (Seville, Spain, 2004). IEEE Automation Congress, Proceedings, 18:251-258 (2004). http://ieeexplore.ieee.org/document/1441050/

2. O. Salvado, C.M. Hillenbrand, D.L Wilson. Int J Biomed Imaging, 2006 (2006). https://www.ncbi.nlm.nih.gov/pmc/articles/PMC2324046/

3. X. Li, M.P. Orchard. IEEE Trans. on Image Processing, 10,10:1521-1527 (2001). http://ieeexplore.ieee.org/document/951537/

4. P. Vandewalle, S. Süsstrunk, M. Vetterli. EURASIP J. Adv. Signal Process, 2006 (2006). https://link.springer.com/article/10.1155/ASP/2006/71459

5. I.K. Somawirata, K. Uchimura, G. Koutaki. IJICIC, 9, 12:4863-4873 (2013). http://www.ijicic.org/ijicic-12-12035.pdf.

6. W. Kim, C. Kim. IEEE Signal Processing Letters, 18, 11:631-634 (2011). http://ieeexplore.ieee.org/document/5986690/?denied

7. S. Avidan, A. Shamir. ACM Trans. Graph., 26,3:267-276, (2007). http://citeseerx.ist.psu.edu/viewdoc/summary?doi=10.1.1.117.7257 
8. S.F. Wang, S.H. Lai. Fast structure-preserving image retargeting, 2009 IEEE International Conference on Acoustics, Speech and Signal Processing, (Taipei, Taiwan, 2009). IEEE ICASSP:1049-1052 (2009). http://ieeexplore.ieee.org/document/4959767/

9. I.K. Somawirata, K. Uchimura, G. Koutaki. Proportional image enlargement using combinations of scaling and carving method, Internationl conference on Signal Image Technology and Internet based System (SITIS), (Kyoto, Japan, 2013). SITIS (2013). http://ieeexplore.ieee.org/document/6727177/

10. I.K. Somawirata, K. Uchimura, G. Koutaki. Image enlargement based on the different scale factors for slice region, The 1st IEEE/ IIAE International Conference on Intelligent Systems and Image Processing (ICISIP), (Kitakyushu, Japan, 2013). ICISIP:119-123 (2013). https://www.researchgate.net/publication/269061963_Image_Enlargement_Based_on_t he_Different_Scale_Factors_for_Slice_Region

11. S. Goferman, L.Z. Manor, A. Tal. EEE Trans. on Pattern Analysis and Machine Intelligence, 34, 10:1915-1926 (2012). http://ieeexplore.ieee.org/document/6112774/

12. I.K. Somawirata, K. Uchimura, G. Koutaki. Image enlargement in different ratio based on the non salient feature enlarged, International Workshop Advanced Image Technology (IWAIT), (Nagoya, Japan, 2013), IWAIT:579-584 (2013). https://scholar.google.co.id/citations?user=c5DnN88AAAAJ\&hl=id 